\title{
Simplicity in Visual Representation: A Semiotic Approach
}

\author{
Ben F. Barton \\ University of Michigan \\ Marthalee S. Barton \\ University of Michigan
}

Simplicity, as an ideal in the design of visual representations, has not received systematic attention. High-level guidelines are too general, and low-level guidelines too ad hoc, too numerous, and too often incompatible, to serve in a particular design situation. This paper reviews notions of visual simplicity in the literature within the analytical framework provided by Charles Morris' communication model, specifically, his trichotomy of communication levels-the syntactic, the semantic, and the pragmatic. Simplicity is ultimately shown to entail the adjudication of incompatibilities both within, and between, levels.

Simplicity, simplicity, simplicity!

H.D. Thoreau

Simplicity as an ideal has a long and venerable history. For the Greeks, the soul was eternal precisely because it was simple. The quest for truth through simplicity led Lucretius to postulate 'atoms' as elemental constituents of matter and Leibnitz to propound 'monads.' Not that simplicity has always been seen in ontological terms: Thoreau's exhortation "Simplify, simplify" was intended as a practical guideline for living. For William James, simplification was a methodology requisite to productive thought:

The facts of the world in their sensible diversity are always before us, but our theoretic need is that they should be conceived in a way that reduces their manifoldness to simplicity.... The simplified result is handled with far less effort than the original data.

Scientific thought rests no less on the ideal of simplicity: According to the Law of Parsimony, the most economical or least complex explanation of a phenomenon is preferred. In design, the ideal of simplicity is epitomized in Mies van der Rohe's famous paradox "Less is more." It is not surprising, then, to encounter simplicity as a general criterion for visual representations. As Albert Biderman observes, "the stereotype of graphics. . is that of an intellectually simple creature. When. . .not simple, they are not. . as ideally supposed to be" (237).

Simplicity as a design goal has, however, recently come under scattered attacks-some broad, some narrow. Architectural theorist Robert Venturi attacks traditional notions of visual simplicity as rooted in the largely discredited modernist design aesthetic epitomized in "Less is more"; according to post-modernist Venturi, "Less 
is a bore." For psychologist Rudolf Arnheim, slavish adherence to the demand for simplicity leads to a lamentable "poverty of abstinence" in visual design. Data analyst John Tukey argues the value of sophisticated graphics for exploring complex data. Similarly, attacking the assumption that data graphics are "mainly devices for showing the obvious to the ignorant" (53), graphics specialist Edward Tufte advocates reserving graphics "for the richer, more complex, more difficult statistical material" (30). The guideline that visuals be simple is challenged by experimental researchers as well: Huntley and Wohlwill ("Children's Responses") find an inverted-U relation between visual complexity and performance; thus, up to a point, performance improves with increasing complexity. Other researchers find that subjects prefer visual complexity (Vitz; Faw and Nunnally; Wohlwill, "Stimulus Exploration").

Should we, then, discard the notion of simplicity as a design ideal? Such a response seems premature. Even considered collectively, the scattered attacks do not constitute a substantive enough basis for rejecting the time-honored ideal of simplicity. They do, however, strongly suggest the need for its re-examination, particularly since the literature reveals no systematic treatment of simplicity in visuals. Moreoever, current guidelines are short on operational utility: Low-level guidelines-e.g., "use no more than four curves on a graph'-are too numerous, too scattered, too ad hoc, too acontextual, and, as we will show, too often incompatible, to be helpful in a particular design situation. On the other hand, high-level guidelines-e.g., 'be simple' - are far too general to effectively inform design practice. As Robert Waller notes: "Complex information can only be made to appear easy and approachable by overlooking the exceptions and the special cases" (284). Barton and Barton lament the lack of an informing analytical framework for graphics design and call for re-validation, with appropriate revisions, of all general guidelines such as the edict for simplicity.

A review of the notions of visual simplicity in the literature is the objective of this paper, which uses the semiotic model of Charles Morris to explore simplicity in terms of three sets of relations:

1. relations of visual elements within a text to each other, whether the text is a single visual, an entire document, a computer display, or a control panel;

2. relations of visual representations to referents, including objects, concepts, and ideas; and

3. relations of visual representations to the conditions of their production and use, including viewer characteristics, purposes, and tasks.

This trichotomy of relations accords generally with the syntactic, semantic, and pragmatic levels, respectively, of Morris' model for human communication. We should note that the model is hierarchi$\mathrm{cal}$, with higher levels subsuming lower ones. Hence, to discuss representation at lower levels in isolation, as we do in sections that 
follow, is to risk oversimplification. The simplification achieved is, however, useful and is ultimately redressed by a subsequent discussion of interactions among levels. The guidelines and references considered are drawn from various fields - including design, graphics, cartography, psychology, computer display, and control theory - for illustrative purposes in an analytical development, with no attempt to be comprehensive.

\section{Syntactic Simplicity}

We begin with notions of simplicity on the syntactic level, the level concerned with relations of visual elements within a text. Because of the focus on internal properties of a visual artifact, the syntactic approach to simplicity is basically formalist, or acontextual. It draws heavily on the field of perceptual psychology, which has emphasized the discovery of universal laws pertaining to human vision. These laws allegedly reveal the intrinsically expressive power of visual elements as perceived by a universalistically conceived viewer. From this perspective, achieving syntactic simplicity entails achieving perceptual simplicity. Stated in the jargon of perceptual psychology, it entails achieving 'figural goodness'; stated in negative, colloquial terms, it entails reducing visual clutter. How does one reduce visual clutter? A review of guidelines and research suggests several methods: limiting the number of both visual elements and variables, and achieving a compatibility of visual elements.

\section{Limitation of the Number of Visual Elements and Variables}

One method of reducing visual clutter is to circumscribe the number of visual elements in a text. Thus, at the document level, authors encounter everywhere the admonition by publishers to hold the number of visuals to an essential minimum (Biderman 232). At the level of the individual visual, one reads, for example, the advice to limit the number of colors since the "use of too many colors can lead to confusion" (Halsey and Chapanis 1057). Similarly, the number of curves in a graph should not exceed four (Woodson and Conover 2-67); elsewhere, the number of segments in a pie chart is limited to five or six (Schmid 65). The specific number of elements recommended in the graphics literature varies and seems based largely on intuition in most cases, but it does accord generally with George Miller's range for the capacity of working memory, i.e., 7 2 "chunks" of information. In contrast, designers of computer displays often appeal directly to Miller's criterion: Thus, Shneiderman advises that the number of alternatives in a menu of computer options should not exceed the limits of working memory.

A second method for reducing visual clutter is to limit the number of visual variables, e.g., size, value, direction, texture, shape, and color. Glynn and DiVesta would circumscribe the number of highlighting cues, including underlining, italics, and fonts, on the basis 
that too many visual cues impede comprehension. Other researchers (e.g., Hartley) warn against the visual cacophony induced by a proliferation of divergent numbering systems within a text, e.g., numbers for pages, chapters, sections, tables, figures, equations, appendices, footnotes, and references.

A related method for reducing visual clutter is to eliminate redundancy. Toward this end, for example, referencing a visual is conventionally deemed preferable to repeating it. Similarly, direct labeling of curves on a graph contributes to syntactic simplicity by obviating the need for a key, hence for a legend. Edward Tufte is among the most radical proponents of syntactic simplicity through removal of redundancy. His analysis of a one-bar chart with inscribed numeric value merits citing in full:

Redundant data-ink depicts the same number over and over. The labeled, shaded bar of the bar chart, for example, unambiguously locates the altitude in six separate ways (any five of the six can be erased and the sixth will still indicate the height): as the (1) height of the left line, (2) height of shading, (3) height of right line, (4) position of top horizontal line, (5) position (not content) of number at bar's top, and (6) the number itself. That is more ways than are needed (96-97).

Elsewhere, Tufte advocates providing range indicators for both dimensions of scatter plots and eliminating the axes as redundant. Moreover, in the presence of such range indicators, even the frames of visuals are deemed redundant and removed. In the field of computer control representation, the elimination of redundancy to simplify the syntax of the command structure was the motivation for Xerox Star's 'generic commands,' e.g., "Show Properties," Star's general mechanism for changing visual properties. According to the designers of the Star workstation, one way to make a system appear simple is to "minimize redundancy.... The ideal system would have a minimum number of powerful commands that achieved all the desired functionality and that did not overlap" (Smith et al. 274).

\section{Compatibility of Visual Elements}

Another approach to syntactic simplicity is to achieve a compatible, or harmonious, relation both among elements of a visual and within a series of visuals. Stated negatively, the goal is to eliminate visual competition, or interference effects. For example, any syntax which precludes perceptual stability should clearly be avoided: The common injunction against graphs with multiple intersecting lines, none of which can readily be traced with certainty, aims at averting such syntactic infelicities. The ease with which textures are created and juxtaposed by computer has given rise to a new admonition: Beware of moiré, or 'op art,' effects. Beyond this, we are urged to enhance the figure/ground relation, e.g., by lightening or eliminating grid lines that compete with data curves, or by inserting space to separate tabulated data into blocks of no more than five entries (Tinker). Other guidelines would enhance the 'goodness' of the figure itself: Schmid suggests that, for reorderable elements, the bars in charts be ordered by increasing length to achieve a perceptually simple trend pattern 
(42); Bertin advocates re-ordering elements of a data matrix for analogous purposes (36). In cartographic design, where outsized visuals are commonplace, concern for figural goodness foregrounds additional perceptual concerns, e.g., limitations of peripheral vision: Figural goodness in large maps is enhanced with a coding scheme that privileges color over size or shape, since color is most distinguishable in peripheral vision (Phillips 166). Similar restrictions are applicable to a variety of engineering diagrams.

Still other guidelines focus on compatibility of syntax among, rather than within, visuals. In this category falls advice to place legends in similar positions on a succession of graphs. In the literature of display design, parallel positioning of elements among multiple panels is standard advice. In control theory, the desired relation between the facility representation and the control display is often couched in terms of syntactic compatibility: In a comparative study of design alternatives, Chapanis and Lockhead conclude that achieving syntactic compatibility between facility representation and control display is far more effective than using interconnecting "sensor lines" between them to indicate correspondences.

But compatibility on a syntactic level is of course, not enough, for surely minimalized visuals and visual elements relate to something besides each other. Surely, for example, they refer to a reality outside themselves, to the external world they are ordinarily taken to represent. To so believe is to enter the realm of the semantic.

\section{Semantic Simplicity}

What, then, is simplicity on the semantic level? The work of John Tukey offers an insightful starting point. Tukey advocates an isomorphism between the complexity of a visual representation and the subtlety of phenomena represented (99; see also Chambers et al.). Semantic simplicity resides, then, not in the natural expressivity of efficiently deployed visual elements but, rather, in the compatibility of a representation with the physical world. In this view, simplicity is not 'artifact-driven,' but is 'data-driven.' Albert Einstein would agree: His assertion that things "should be made as simple as possible, but not simpler" reflects a belief in the concordance of scientific representation with the very nature of the physical world. Not surprisingly, Einstein's endorsement accords generally with the positivistic philosophical outlook prevailing in science. Even less surprisingly, this positivistic view of science permeates the thinking of non-scientists as well: Ashwin, for example, sees the degree to which visuals are data-driven as the distinctive difference between fine art and technical illustration (60). In the following paragraphs, semantic simplicity is related to a compatibility of referent and representation, including its format and visual variables.

\section{Compatibility of Referent and Representation}

In the graphics literature, the idea of representation as referent- or data-driven is usually presented in a reductionist form; that is, there is a belief in the compatibility of referent and representation, but the 
referent is thought singular in meaning. This idea is expressed widely in the literature as the design goal of 'one idea per visual.' Jacques Bertin's definition of graphics as "a monosemic [unique-meaning] system" (2) merely casts the ideal into semiotic vocabulary. The reductionistic view of semantic simplicity has long dominated the design of visual displays in the control field: Witness the hegemony there of the 'one measurement-one indication' approach, the technical version of 'one idea per visual' (Goodstein). The goal of semantic simplicity also led the designers of Xerox Star to augment the traditional typewriter keyboard with peripheral, special-purpose 'function keys,' now standard on workstations. This strategy avoided assigning multiple meanings to Star keys, whereas keys were used both to enter alphanumerics and to invoke commands on the earlier Bravo workstation. Rubinstein and Hersh offer a general guideline corroborating the approach of the Star designers: "Multiplexing of function-using the same input for more than one purpose-is usually poor in terms of human performance and should be severely limited" (47). The literature of the graphics-communication field also offers guidelines for constructing monosemic visual representations. Bertin suggests choosing a "preferred question" and constructing a visual biased to allow readers to easily answer it, even if such biasing requires sacrificing information (12). In a similar vein, Kathryn Szoka advises formulating a "focus sentence" summarizing the point of the visual as the first step in graph design (101).

\section{Compatibility of Referent and Representation Format}

Semantic compatibility, broadly discussed so far, clearly entails subsidiary compatibilities-most notably, a compatibility between a referent and the format of the representation. The broadest distinction made in the literature is that between symbolic and figural representations, often expressed in terms of digital vs. analog representations, respectively. Wickens argues that some physical attributes are apprehended "naturally" in one or the other of these formats, and that designers should not violate these "natural" associations (178-80). He contends, for example, that altitude is an analog concept-thought to take on values in a continuum - and hence should be represented in analog format, e.g., with a moving needle, rather than in digital format, e.g., with a numeric indicator. The view of semantic simplicity as a compatibility of referent and format is implicit in the genre-based approaches to visual design endemic in the literature of technical and business communication. Thus, it underlies assertions of the general form 'genre best displays phenomenon' - e.g., 'line graphs best display trends in the data.' In this case, the reasoning is roughly that a trend implies directionality and only an (abstract) line is uniquely characterized by the directional attribute (Schutz). Reporting an experimental confirmation of this guideline, Schutz speculates that vertical bars in charts impede the reading of trends because their vertical lines arrest eye movement. His reasoning rests firmly on the principle of semantic compatibility of referent and representation format. 


\section{Compatibility of Referent and Visual Variables}

Semantic simplicity also requires a compatibility of referent and visual variables. In this connection, we further consider altitude as an analog concept; Wickens discusses the referential compatibilities, in an altimeter display, of three visual variables, namely, shape, direction, and dimensionality (180). Shape compatibility suggests a linear rather than, say, a circular display. (A circular display might, on the other hand, be appropriate for an indicator of compass heading.) Similarly, directional compatibility dictates a vertical display with high values on top rather than, say, a horizontal display with high values on the right-or, which is worse, on the left. Dimensional compatibility suggests the use of a single pointer rather than, say, three pointers serving in the interrelated manner of clock hands, since altitude is regarded as a unitary concept. Concern for dimensional compatibility also underlies Edward Tufte's recommendation that "the number of information-carrying (variable) dimensions should not exceed the number of dimensions in the data" (71; see also Grether and Baker, 77). On these grounds he would, for example, forbid the use of three-dimensional bars in a (twodimensional) graph.

Semantic compatibility governs the handling of other visual variables as well. Textural compatibility underlies the conventional use in graphs of solid lines to represent empirical values and dashed lines to represent projected, or extrapolated, values. The fact that dashed lines are less substantial than solid lines mirrors the fact that projected values are less certain than empirical ones. Similar reasoning holds for textural compatibility achieved through the use of heavier lines for data curves than for grids or axes, though here semantic compatibility depends on the relative importance, rather than on the relative certainty, of the data. Semantic compatibility also governs the choice of size and placement of visual elements. Thus, we are advised to make important things larger, as in exploded views, and centrally rather than peripherally located in a visual (Olson and Huckin 129-30). Moreover, semantic compatibility governs intervariable choices, as well as the intravariable choices just discussed. Thus, gray scales rather than colors are recommended for representing quantitative phenomena, such as population density, on the basis that colors are not apprehended as ordered, e.g., relative to wavelength (Bertin 87).

A review of the preceding paragraphs reveals that the viewer's role in assigning meaning has slowly been made more explicit. And it must be admitted, after all, that there can be no meaning without a viewer. The posited viewer, however, remains universalisticthough now concerned with meaning rather than 'pure form'-and, as such, washes out as a variable. But what happens when the viewer is seen not as a universal but as a variable, and a central variable at that? What happens, for instance, when a challenge is thrown up to Wickens' claim that some variables are apprehended as analogical in their very nature? What happens, in other words, if the distinction between analog and digital is seen as conventional rather than natu- 
ral? Clearly, semantic simplicity eventually raises, but ultimately cannot answer, such questions. For answers we must turn to the realm of pragmatic simplicity, the focus of the following section.

\section{Pragmatic Simplicity}

The pragmatic level of Morris' model encompasses the situational, or contextual, specifics in communication. It thus includes constraints in the production and use of a visual representation. Simplicity on the pragmatic level entails a compatibility of representations with both viewer and production requirements.

\section{Compatibility of Representation and Viewer Requirements}

We begin with the viewer, for whom perception has become a constructive, rather than a receptive, act. Representations, then, are no longer artifact- or data-driven, but viewer-driven. Simplicity is now in the eye of the beholder and, as film theorist Gavriel Salomon argues, "The more congruent the symbolic carriers of a message are with one's specific symbolic mode of internal representation the less translation is needed, and the more information will be extracted and presumably learned" (218). But this congruity, as Salomon hastens to add, is not a simple matter, for the viewer is now seen as a multiplicity rather than a monolith and notions of simplicity multiply accordingly. Danko Plevnik applauds this proliferation of simplicities: In an article directly critical of "outdated concepts" of simplicity in verbal materials, he advocates a pluralistic notion of simplicity rooted in user needs. How pluralistic? In the pragmatic view, the extreme of either a universalistic or an idiosyncratic viewer is indefensible. Pragmatism posits a via media between the two extremes through the notion of user classes based on shared conventions. Pragmatic simplicity dictates, for example, that visual representations be compatible with cultural stereotypes. Brandt's research suggests the virtue of layouts compatible with scanning patterns used in reading. In Western societies, this suggests a left-toright disposition of visual materials. On the other hand, one expects this disposition to create problems for viewers with a different reading pattern, e.g., the Japanese.

Similarly, Osborne et al. emphasize that display colors should accord with "general population stereotypes." Their criticism of the color-coding schemes in some plants is an implicit endorsement of the compatibility principle: Noting that in many Western societies red $=$ danger, yellow $=$ caution, and green $=$ normal, they lament a violation in which green indicates an open valve and red a closed one, producing a 'Christmas tree' impression in normal operationwhen some valves are open, others closed-that may not be distinguishable at a glance from the appearance at system failure. Osborne et al. opt for a code in which green indicates the 'normal' valve position, and red the 'abnormal' position, whether open or 
closed. With such a display code, an abnormality shows up as a salient red indicator against a green background. Conventions in a different culture may dictate a different coding system: Courtney documents the fact that the color associations of the Chinese differ strongly from those of Americans: For example, Chinese subjects most often chose green for 'on,' whereas Americans are more likely to choose red. Conventions are also associated with disciplines and vary among them: A mechanical engineer codes with red a 'closed' valve, meaning that it is not allowing flow. On the other hand, an electrical engineer uses a diode that emits red light when 'closed,' meaning that it is allowing (electrical current) flow.

Pragmatic simplicity is also a product of experience and training. Lusk and Kersnick find that "when individuals are asked to render perceived complexity judgments, the reports which are similar in nature to the report with which they are familiar are likely to be ranked as not complex" (797). Individuals prefer reports "similar to those for which they have developed operationally valid heuristics" (797). Cartographer Thorndyke applies the principle of compatibility of representation and user experience to the problem of map design. In his discussion of alternative navigational aids for users with an unfamiliar itinerary, he advocates ego-centered frame-ofreference ('route') maps-exemplified in the familiar AAA 'strip' maps-for lay users. On the other hand, he endorses traditional, 'World-oriented' ('North-up') maps for experts, who gain from their ability to use greater representational complexity if the need arises to improvise a path in the face of disruptive circumstances.

In the quest for pragmatic simplicity, perhaps no compatibility is more critical than that between a visual representation and the viewer's task. The recurrent question whether to display data in graphic or tabular form, for example, may be resolved through an appeal to the task-compatibility principle. Experimental research into the relative superiority of graphic and tabular representations confirms the need for representation-task (or, in psychological jargon, 'stimulus-response') compatibility. The studies of both Washburne, and Lusk and Kersnick, indicate that tables are superior to graphics for subjects asked to look up and recall specific values. Similarly, Vernon finds tables superior to graphics for subjects required to comprehend demographic statistics. Conversely, Feliciano et al. find graphics superior to tables for subjects asked to compare data. On the other hand, Powers et al. finds combinations of text and graphics superior for subjects required to make decisions when accuracy is paramount. Pragmatic simplicity may also govern the choice between a series of syntactically simple graphs and a single complex composite visual, for data representation. For example, Schutz finds that, while separately plotted graphs are serviceable for reading data values, superimposed graphs are significantly superior for comparing data.

Pragmatic simplicity also dictates that representations be compatible with the environment in which tasks are performed. The 
massive experimental studies by Dwyer ("Program"; "Strategies") and his associates confirm the validity of such a compatibility: They conclude that, while simplified line drawings are best for fixed-pace media instruction, realistic photographs are best for learner-paced instruction where the viewers have time to examine detail. Some guidelines in the graphics literature tacitly recognize the influence of task environment: Thus, we read that visuals for oral presentations should be simpler than their counterparts in written texts, since the viewer generally has less time to examine them (Michaelson 154).

\section{Compatibility of Representation and Production Requirements}

Turning from conditions of use to limits of production, we note that some designers regard the latter as paramount. Jan Tschichold, for example, makes compatibility with capabilities of the printing press one of only two criteria in the typographic design of books. In any case, it is clear that the means of production limit attainable representational complexity: Susan Star observes that equipment limitations, e.g., the number of channels in monitoring equipment, often unduly restrict the complexity of representations in biological research. Similarly, difficulties in the hand-execution of visuals once led technical communicators to underuse such potentially powerful, easy-to-grasp genres as pictographs, much praised by graphics specialists, e.g., Neurath and Macdonald-Ross. But production limitations change and, with them, notions of pragmatic simplicity. Clearly, computers have altered the prevailing notions of what is, or is not, simple to execute: Bit-mapped graphics systems now permit a wide variety of pictographic elements to be easily defined and manipulated by a lay illustrator. Pictographs are, in fact, enjoying a revival in the representation of queuing problems. Not that the influence of the computer as a facilitator of graphics has been uniformly beneficent: We have discussed the facile use of extraneous elements in the computer-generated graphics of both students and professionals (137); Edward Tufte provides ocular proof of such graphic gratuities in his generously illustrated chapter on "chartjunk."

\section{The Problem of Incompatibilities}

We have seen that simplicity in visual representation entails numerous compatibilities on each of three levels-the syntactic, the semantic, and the pragmatic. This is not to suggest that the design of simple visuals is simple for, unfortunately, the compatibilities discussed are not necessarily mutual compatibilities. Our present purpose, in fact, is to illustrate the gamut of incompatibilities that may arise, both within and between levels, in the design of a representation-incompabilities that imperil the attainment of visual simplicity. 


\section{Incompabilities within Levels}

\section{Syntactic/Syntactic}

Consider, first, incompatibilities at the syntactic level. We have already discussed the compatibility of syntax among elements of a visual and, subsequently, the compatibility of syntax among visuals; here, we address the problem of incompatiblities between these two syntaxes. Consider the potential for syntactic incompatibilities in a series of pie charts, for example. Guidelines for syntactic simplicity within a visual dictate ordering segments by size; suppose this yields the pattern $A B C D$ on an initial chart. Now, application of the same guidelines to a second chart with different relative values may yield a different segment pattern, say CADB. Guidelines for syntactic simplicity among visuals, however, dictate that the segments of the two charts manifest the same order, i.e., ABCD. An incompatibility has arisen. The controversy over the desirability of right-justifying text may also be framed in terms of competing syntactic requirements. Right justification achieves the syntactic simplicity associated with the figural goodness of the page as a whole; however, the attendant erratic spacing between the letters of a word degrades the figural goodness of the word considered as an image. The recommendation against right justification increasingly found in recent literature signals the triumph of syntactic simplicity at the word level. The literature on control display shows a strong cognizance of the problem of adjudicating such syntactic incompatibilities. For multiple-panel displays, particularly those with substantial variations in intra-panel syntaxes, the choice of an inter-panel syntax is an endemic problem. The choice is critical in, say, control systems for nuclear-power plants, where operators may confront hundreds of panels and thousands of meters in crisis situations (Seminara et al.; Goodstein; Mitchell).

\section{Semantic/Semantic}

A semantic incompatibility occurs when a visual representation may be invested with multiple meanings. A classic example occurs in the representation of data with scaled replicas of two- or threedimensional entities. With representations using two-dimensional entities, e.g., circles or dollar bills, meaning may be invested either in a linear dimension or in the area of the figure. Thus, a doubling of all figural dimensions permits two different interpretations: a doubling of the value represented under the first interpretation, but a quadrupling of the value under the second. Similarly, three different interpretations result from investing meaning in a linear dimension, in the area, or in the implied volume of representations of threedimensional entities, e.g., oil barrels, animals, or buildings. A broad class of potential semantic incompatibilities involves representations of several phenomena plotted on a single chart or graph. Such a 
composite representation may foster unwarranted inferences of causal relations among the phenomena, particularly if the representational patterns happen to show either strong positive or negative correlations. Caveat spectator! A bar chart representing changes in two phenomena may be compared to a work of art in which strips of two representations appear alternately, so that each of the representations functions as a 'venetian blind' for the other. In either case, viewers may be prone to find a relation between the two phenomena represented, whether warranted or not. We can only mention distortion in graphics, another broad area involving semantic incompatiblities. Semantic incompatibilities are, of course, irreconcilable with the monosemic view of graphics discussed earlier.

\section{Pragmatic/Pragmatic}

Incompatibilities at the pragmatic level are, perhaps, most easily understood. Whenever a given visual representation is destined for different classes of users or for different tasks, pragmatic incompatibilities are inevitable. Recalling our earlier discussion of color associations, how does one devise a color-coding scheme for a software manual that must serve both American and Chinese viewers? In the area of computer display, a well-known example occurs when both novices and experts confront command menus. Novices benefit from a menu presentation that displays available options, a memory-support aid unnecessary for the expert. For the expert, who has long since committed the list to long-term memory, the menu list is visual clutter (Wickens 236). Clearly, simplicity is now in the eye of the beholder.

Different task demands also lead to pragmatic incompatibilities. A classic example from cartography involves the use of a common representation both for navigating and for estimating flying times. Navigating requires greater detail in more heavily populated or land-marked areas, whereas accurately estimating flying time is impeded by non-uniformity of map texture. Again, consider the implications of default or automatic features of computer-graphics systems. Aimed at simplicity of production, they are, unfortunately, not always compatible with representational requirements in a given situation: Automatic exploding of the largest segment of a pie chart facilitates production but may highlight an inappropriate segment for the viewer.

\section{Incompatibilities between Levels}

\section{Syntactic/Semantic}

Incompatibilities imperiling visual simplicity may also arise between levels. Consider, first, incompatibilities between the syntactic and the semantic levels. Such incompatibilities are especially prevalent in highly mimetic visual genres, e.g., genres with strong links to the physical world, like photographs or maps. Cartographer Richard Phillips notes the chronic difficulty of achieving syntactic simplicity 
in representing entities which lack figural goodness, e.g., woodland areas. Evelyn Goldsmith, who would understand Phillips' quandary, offers another example of a chronic syntactic/semantic incompatibility: She notes that insofar as (syntactic) complexity has an attraction value independent of any meaning, there is always a potential area for conflict with the meaning, or semantic, level (278).

Not that syntactic/semantic incompatibilities always remain unresolved, or open, issues. In fact, some genres may be considered embodiments of de facto, conventionalized resolutions of these conflicts, e.g., exploded views and 'value-by-area' maps. Consider the case of the exploded view. Syntactic simplicity dictates the use of a uniform scale over a representation; semantic simplicity, on the other hand, dictates that important things be made larger, thereby validating the use of two scales. The exploded-view convention thus amounts to an adjudication in favor of semantic simplicity at the expense of syntactic simplicity. Such exceptions aside, syntactic/ semantic incompatibilities remain to be negotiated in the design of a given visual.

\section{Semantic/Pragmatic}

Consider, next, incompatibilities between requirements at the semantic and pragmatic levels. Many of them originate in conventions springing from the production limitations of earlier technologies. Garland notes the continuing domination of the "rectilinear mode," omnipresent in the era of the stencil and the straightedge. Especially lamentable is the persistence of the "ubiquitous oblong box" in graphic representations of computing concepts; something which may mean almost anything means nothing. Noting the "remarkable range of items and ideas [e.g., process, attribute, event, entity, selection, step or operation] which oblongs and oblong derivatives are currently used to represent" (176), Entwhistle advocates more expressive, varied representations. Fortunately, the means for resolving this incompatibility is already at hand for, as Entwhistle notes, "we need not be confined to the stencil, nor to geometric simplicity" (177): The computer can readily generate a wide variety of forms more suggestive of the many meanings now invested in the ubiquitous oblong box.

Not that the computer automatically solves every semantic/ pragmatic incompatibility. Machines have limitations in every era, and there are always potential problems in achieving semantic simplicity: Today, for example, some workstations do not provide for variable line thickness in graphs, a limitation that precludes the expressive use of line width as a meaning-bearing visual variable.

\section{Syntactic/Pragmatic}

Last, consider incompatibilities between requirements at the syntactic and pragmatic levels. The handling of redundancy in visual representations readily comes to mind. Syntactic simplicity dictates avoidance of redundancy; pragmatic simplicity may dictate the op- 
posite. The publication policy of handling repeated needs for a visual by referral rather than repetition represents an adjudication in favor of syntactic simplicity. On the other hand, Hartley's advice to duplicate headings on the left and right sides of wide tables for ease of lookup is a verdict in favor of pragmatic simplicity. As Patricia Wright sensibly observes, "Eliminating redundancy can be a false economy" (337).

Not that adjudications of syntactic/pragmatic incompatibilities are always so conscious in visual design. A dramatic example of the neglect of pragmatic simplicity occurred in the design of a symbol system based primarily on the ideal of syntactic simplicity: Lodding discusses "airport signs, circa 1972, which were designed to indicate departing and arriving flights" (14). Simplification of the syntax for the sign system led to the use of airplane silhouettes aimed obliquely upward for departing planes and obliquely downward for arriving planes. But the simple syntax of the symbol pair had to be abandoned when it was soon discovered that passengers associated the symbol for arriving airplanes with an impending crash.

Nor has the recognition of syntactic/pragmatic incompatibilities always led to so swift a resolution in practice. Here is Josef Albers bemoaning the continuing widespread use of sans-serif, rather than serif, typefaces in the face of mounting evidence of incompatibility at the pragmatic level:

The concept that "the simpler the form of a letter the simpler its reading" was an obsession of beginning constructivism. It became something like a dogma and is still followed by "modernistic" typographers... Ophthalmology has disclosed that the more the letters are differentiated from each other, the easier is the reading. Without going into comparisons and details, it should be realized that words consisting of only capital letters present the most difficult reading-because of their equal height, equal volume, and, with most, their equal width. When comparing serif letters with sans-serif, the latter provide an uneasy reading. The fashionable preference for sans-serif in text shows neither historical nor practical competence (4).

For Albers, resolving the incompatibility between the syntactic simplicity of sans-serif letters and the pragmatic simplicity of serif letters was easy. He already knew what the airport symbol-system designers had to discover: Pragmatic simplicity-the overarching consideration in Morris' model-must prevail. More typically, however, inter-level incompatibilities are not so easily adjudicated. David Smith et al. of the Xerox Corporation discuss their difficulties in settling on a display format for the mouse controller of the Star workstation. Led by a desire for syntactic simplicity to initially propose a one-button mouse as an alternative to the three-button mouse then in vogue, the designers soon discovered that "what is simplest along any one dimension [e.g., number of buttons] is not necessarily conceptually simplest for users" (276). To their surprise, extensive experimentation with users established the clear superiority of a two-button mouse. Thus, the final design rested on a compromise 
between the syntactic simplicity of the one-button format and the functional simplicity of the three-button format.

\section{Conclusion}

Let us return to our original question: Should we discard the notion of simplicity as an ideal in visual design? Our analysis leads us to answer "No." Visual design is a problem-solving process, and problem analysis is an essential first step. Morris' model fosters an analytical approach offering more promise than the asystematic application of peremptory guidelines whose theoretical bases have been obscure. Admittedly, reconciling often conflicting requirements in pursuit of a multiplicity of simplicities is not easy. But design never is. As industrial designer David Pye astutely observes, all practical designs "are in some degree failures, either because they flout one or another of the requirements or because they are compromises, and compromise implies a degree of failure" (77). If there are no ideal solutions, it is, nonetheless, possible to determine provisionally the best available accommodation in a given design situation. And that's the simplest a visual representation should be.

\section{References}

Albers, Josef. Interaction of Color. Rev. ed. New Haven: Yale University Press, 1975.

Arnheim, Rudolf. Visual Thinking. Berkeley and Los Angeles: University of California Press, 1969.

Ashwin, Clive. "The Ingredients of Style in Contemporary Illustration: A Case Study." Information Design Journal 1(1979): 51-67.

Barton, Ben F. and Marthalee S. Barton. "Toward a Rhetoric of Visuals for the Computer Era." The Technical Writing Teacher 12.2(1985): 126-45.

Bertin, Jacques. Semiology of Graphics: Diagrams, Networks, Maps. Trans. William J. Berg. Madison, Wisc.: University of Wisconsin Press, 1983.

Biderman, Albert D. "The Graph as a Victim of Adverse Discrimination and Segregation: Comment Occasioned by the First Issue of Information Design Journal." Information Design Journal 1.4(1980): 232-41.

Brandt, Herman F. The Psychology of Seeing. New York: The Philosophical Library, 1945.

Chambers, John M. et al. Graphical Methods for Data Analysis. Boston: Duxbury Press, 1983.

Chapanis, Alphonse and G. Lockhead. "A Test of the Effectiveness of Sensor Lines Showing Linkages Between Displays and Controls." Human Factors 7.3(1965): 219-29.

Courtney, Alan J. "Chinese Population Stereotypes: Color Associations." Human Factors 28.1(1986): 97-99.

Dwyer, Francis M. "The Program of Systematic Evaluation: A Brief Review." International Journal of Instructional Media 10(1982-83): 23-38.

Strategies for Improving Visual Learning: A Handbook for the Effective Selection, Design, and Use of Visualized Materials. State College, Penn.: Learning Services, 1978.

Entwhistle, David. "The Use of Graphics In Computing: A Personal View 
From the Inside." Information Design Journal 3.3(1983): 173-82.

Faw, Terry T. and Jum C. Nunnally. "The Influence of Stimulus Complexity, Novelty and Affective Value on Children's Fixations." Journal of Experimental Child Psychology 15(1973): 488-99.

Feliciano, Gloria D., Richard D. Powers, and Bryant E. Kearl. "The Presentation of Statistical Information." Audio-Visual Communication Review (ECTJ) 11.3(1963): 32-39.

Garland, Ken. "Some General Characteristics Present in Diagrams Denoting Activity, Event and Relationship." Information Design Journal 1.1(1979): 15-22.

Glynn, Shawn M. and Francis J. DiVesta. "Control of Prose Processing via Instructional and Typographic Cues." Journal of Educational Psychology 71(1979): 595-603.

Goldsmith, Evelyn. Research Into Illustration: An Approach and a Review. Cambridge: Cambridge University Press, 1984.

Goodstein, Larry P. "Discriminative Display Support for Process Operators." In Human Detection and Diagnosis of System Failures. Eds. Jens Rasmussen and William B. Rouse. New York: Plenum, 1981. 433-49.

Grether, Walter F. and C. A. Baker. "Visual Presentation of Information." In Human Engineering Guide. Eds. H.P. Van Cott and R.G. Kinkade. Washington: U.S. Government Printing Office, 1972. 41-121.

Halsey, R. M. and Alphonse Chapanis. "On the Number of Absolutely Identifiable Spectral Hues." Journal of the Optical Society of America 41(1951): 1057-58.

Hartley, James. Designing Instructional Text. London: Kogan Page, 1978.

Hartley, James et al. "The Role of Spatial and Typographic Cues in the Layout of Journal References." IEEE Transactions on Professional Communication PC-23.3 (1980): 138-42.

Huntley, M. Stephen. "Task Complexity and Serial Performance under Steadily Increasing Input Rates." Human Factors 14.1(1972): 65-72.

James, William. "The Sentiment of Rationality." In Essays in Pragmatism. New York: Hafner, 1948. 3-36.

Lodding, Kenneth N. "Iconic Interfacing." IEEE Computer Graphics and Applications 3.2(1983): 11-20.

Lusk, Edward J. and Michael Kersnick. "The Effect of Cognitive Style and Report Format on Task Performance: The MIS Design Consequences." Management Science 25.8(1979): 787-98.

Macdonald-Ross, Michael. "How Numbers Are Shown: A Review of Research on the Presentation of Quantitative Data in Texts." Audio-Visual Communication Review (ECTJ) 25.4(1977): 359-409.

Michaelson, Herbert B. How to Write and Publish Engineering Papers and Reports. 2nd ed. Philadelphia: iSi Press, 1986.

Miller, George A. "The Magical Number Seven, Plus or Minus Two: Some Limits on Our Capacity for Processing Information." Psychological Review 63.2(1956): 81-97.

Mitchell, Christine M. and R. A. Miller. "Design Strategies for ComputerBased Information Display in Real-Time Control Systems." Human Factors 25.4(1983): 353-69.

Morris, Charles. Foundations of the Theory of Signs. Chicago: University of Chicago Press, 1938. 
Neurath, Marie. “Isotype." Instructional Science 3(1974): 127-50.

Olson, Leslie A. and Thomas N. Huckin. Principles of Communication for Science and Technology. New York: McGraw-Hill, 1983.

Osborne, P. D., H. F. Barsam and D. C. Burgy. "Human Factors Considerations for Implementation of a 'Green Board' Concept in an Existing 'Red/Green' Power Plant Control Room." In Proceedings, 25th Annual Meeting of the Human Factors Society. Ed. R. C. Sugarman. Santa Monica: Human Factors Society, 1981.

Phillips, Richard J. "Making Maps Easy to Read: A Summary of Research." In Processing of Visible Language: Vol. 1. Eds. Paul A. Kolers, Merald E. Wrolstad and Herman Bouma. New York: Plenum, 1979. 165-74.

Plevnik, Danko. "The Implicity of Simplicity: Toward the Theory of Individual Information." Information Design Journal 4.1(1984): 19-24.

Powers, Matthew, Conda Lashley, Pamela Sanchez and Ben Shneiderman. "An Experimental Comparison of Tabular and Graphic Data Presentation." International Journal of Man-Machine Studies 20(1984): 545-66.

Pye, David. The Nature of Design. London: Studio Vista and New York: Van Nostrand Reinhold, 1964.

Rubinstein, Richard and Harry M. Hersh. The Human Factor: Designing Computer Systems for People. Burlington, Mass.: Digital Press, 1984.

Salomon, Gavriel. Interaction of Media, Cognition, and Learning: An Exploration of How Symbolic Forms Cultivate Mental Skills and Affect Knowledge Acquisition. San Francisco: Jossey-Bass Publishers, 1979.

Schmid, Calvin F. Statistical Graphics: Design Principles and Practices. New York: John Wiley and Sons (Wiley-Interscience), 1983.

Schutz, Howard G. "An Evaluation of Methods for Presentation of Graphic Multiple Trends: Experiment III." Human Factors 3(1961): 108-19.

Seminara, Joseph L., S. Eckert, S. Seidenstein, W. Gonzalez, R. Stempson and S. Parsons. Human Factors Methods for Nuclear Control Room Design. EPRI NP-1118-SY. Washington: Electric Power Research Institute, 1979.

Shneiderman, Ben L. Software Psychology. Cambridge, Mass.: Winthrop, 1980.

Smith, David C., Charles Irby, Ralph Kimball, Bill Verplank and Eric Harslem. "Designing the Star User Interface." Byte 7(1982): 242-82.

Star, Susan L. "Simplification in Scientific Work: An Example from Neuroscience Research." Social Studies of Science 13(1983), 205-28.

Szoka, Kathryn. "A Guide to Choosing the Right Chart Type." IEEE Transactions on Professional Communication PC-25.2(1982): 98-101.

Thoreau, Henry D. Walden and Civil Disobedience. Ed. Owen Thomas. New York: Norton, 1966.

Thorndyke, Perry W. Performance Models for Spatial and Locational Cognition. Technical Report R-2676-ONR. Washington: Rand Corporation, 1980.

Tinker, Miles A. Bases for Effective Reading. Minneapolis: University of Minnesota Press, 1965.

Tschichold, Jan. Asymmetric Typography. New York: Reinhold Publishing Company, 1967.

Tufte, Edward R. The Visual Display of Quantitative Information. Cheshire, Conn.: Graphics Press, 1983. 
Tukey, John W. Exploratory Data Analysis. Reading, Mass.: AddisonWesley, 1977.

Venturi, Robert. Complexity and Contradiction in Architecture. New York: The Museum of Modern Art, 1966.

Vernon, Magdalen D. "Learning from Graphical Material." British Journal of Psychology: General 36.3(1946): 145-58.

Vitz, Paul C. "Preference for Different Amounts of Visual Complexity." Behavioral Science 11(1966): 105-14.

Waller, Robert H. W. "Review of Tom Vernon's Gobbledegook." Information Design Journal 1.4(1980): 283-85.

Washburne, John N. “An Experimental Study of Various Graphic, Tabular and Textual Methods of Presenting Quantitative Material." Journal of Educational Psychology 18.6(1927): 361-76 and 465-76.

Wickens, Christopher D. Engineering Psychology and Human Performance. Columbus: Charles E. Merrill Publishing Co., 1984.

Wohlwill, Joachim F. "Amount of Stimulus Exploration and Preference as Differential Functions of Stimulus Complexity." Perception and Psychophysics 4(1968): 307-12.

"Children's Responses to Meaningful Pictures Varying in Diversity: Exploration Time vs. Preference." Journal of Experimental Child Psy chology 20(1975): 341-55.

Woodson, Wesley and Donald W. Conover. Human Engineering Guide for Equipment Designers. 2nd ed. Berkeley and Los Angeles: University of California Press, 1966.

Wright, Patricia. "A User-Oriented Approach to the Design of Tables and Flowcharts," In The Technology of Text: Principles for Structuring, Designing, and Displaying Text. Ed. David H. Jonassen. Englewood Cliffs, N.J.: Educational Technology Publications, 1982. 317-39. 